Revista Brasileira de Agricultura Irrigada v.2, n.1, p.24-28, 2008

ISSN 1982-7679 (On-line)

Fortaleza, CE, INOVAGRI - http://www.inovagri.org.br

Protocolo 002.07 - 23/09/2007 Aprovado em 25/03/2008

\title{
COLETOR AUTOMÁTICO PARA ENSAIO DE ASPERSORES EM LABORATÓRIO (1) - DESENVOLVIMENTO DO MODELO
}

TADEU MIRANDA DE QUEIROZ¹, SÍLVIO CARLOS RIBEIRO VIEIRA LIMA²,

$$
\text { TARLEI ARRIEL BOTREL }{ }^{3} \text {, JOSÉ ANTÔNIO FRIZZONE }{ }^{4}
$$

\footnotetext{
${ }^{1}$ Eng. Agrícola, Doutor, Prof. Prof. Assistente UNEMAT e-mail: tdmqueiroz@yahoo.com.br

${ }^{2}$ Eng. Agrônomo, Mestre, Prof. FATEC - CENTEC Sobral, Doutorando ESALQ/USP

${ }^{3}$ Eng. Agrônomo, Doutor, Prof. Associado ESALQ/USP email:tabotrel@esalq.usp.br

${ }^{4}$ Eng. Agrônomo, Doutor, Prof. Titular ESALQ/USP email:frizzone@esalq.usp.br
}

RESUMO: O desenvolvimento de um coletor para precipitação de aspersores em laboratório foi alvo de estudo para implantação no Laboratório de Ensaios em Equipamentos de Irrigação - LEEI, em SobralCe. Foram estudados os melhores formatos para a coleta da precipitação, levando em conta os fatores: volume coletado, material utilizado e adaptação à automação utilizando sensores de pressão como forma mais econômica de utilização. Conclui-se que os coletores aqui desenvolvidos possuem plena capacidade de serem utilizados para os ensaios e que devem ser realizados testes para confirmar os estudos.

Palavras-chave: aspersão, coletor, automação

\section{DEVELOPMENT OF THE AUTOMATIC COLLECTOR FOR TESTING OF SPRINKLERS IN LABORATORY}

ABSTRACT: The development of a collector for precipitation of the sprinklers in laboratory was studied for implantation at the Laboratory of Testing in Equipments of Irrigation - LEEI. They were studied the best format for the collection of the precipitation, taking into account the factors collected volume, used material and adaptation the automation using sensor of pressure as more economical form of use. It is ended that the collectors possess full capacity of they be used for the testing and tests should be accomplished to confirm the studies.

Keywords: sprinkler, collector, automation

\footnotetext{
${ }^{1}$ Eng. Agrícola, Doutor, Prof. Prof. Assistente UNEMAT e-mail: tdmqueiroz@yahoo.com.br

${ }^{2}$ Eng. Agrônomo, Mestre, Prof. FATEC - CENTEC Sobral, Doutorando ESALQ/USP

${ }^{3}$ Eng. Agrônomo, Doutor, Prof. Associado ESALQ/USP email:tabotrel@esalq.usp.br

${ }^{4}$ Eng. Agrônomo, Doutor, Prof. Titular ESALQ/USP email:frizzone@esalq.usp.br
}

R. Bras. Agric. Irrigada v.2, n.1, p.24-28, 2008. 
Miranda de Queiroz et al.

\section{INTRODUÇÃO}

De acordo com Lima (2001) o Brasil é carente em ensaios de equipamentos de irrigação, pois como grande entrave para esta situação é a deficiência de laboratórios específicos e corpo técnico qualificado a realizar estes ensaios. Quando falamos de ensaio em laboratórios, devemos nos preocupar com a confiabilidade dos dados, com a metodologia utilizada e, principalmente, com as inovações tecnológicas, visando uma maior facilidade para a realização de ensaios em laboratórios de instituições científicas e tecnológicas que trabalham com irrigação, o que certamente resultará em um maior desenvolvimento tecnológico do setor.

Em se tratando de ensaios de aspersores em laboratório, o circuito de coletores é um equipamento indispensável para a determinação do perfil de distribuição, o que é um dos requisitos previstos previsto por ABNT (2000).

O design de coletores já foi estudado por vários autores como Kohl (1972) e Neff (1977). Marek et al. (1985) avaliou diferentes tipos de coletores comparados com uma referência, usando intensidades de chuva diferentes e níveis diferentes de demanda de evaporação. Carran (1976) mostrou que o erro de medida de precipitação devido à radiação solar é significativo. Hendawi et al. (2002), cita que o diâmetro da área do coletor é selecionado de acordo com o tempo de ensaio; pois quanto mais longo for o tempo, menor será o diâmetro. Este autor cita o trabalho de Fischer et al. (1988), onde aquele autor sugeriu que o diâmetro do coletor deveria ser de $40 \mathrm{~mm}$ quando a duração do ensaio sem vento fosse de 120 minutos e de $235 \mathrm{~mm}$ para 15 minutos de ensaio. $\mathrm{O}$ efeito de um coletor cilíndrico em condições de fluxo de ar e suas consequiências na coleta de água foi avaliado experimentalmente em um túnel de vento por Mueller et al. (1972) e posteriormente por Livingston et al (1985). Eles concluíram que o erro na medida excede $20 \%$ para ventos maiores que $5 \mathrm{~m} / \mathrm{s}$, piorando com a altura do coletor, com o diâmetro e com diminuições no tamanho da gota.

De acordo com Hendawi et al. (2002), tendo a variabilidade de condições de clima como fator decisivo, pode-se concluir que a forma de coletor ideal outdoor não existe. Este autor testou com simulações numéricas de corrente de ar ao redor de coletores de formas diferentes e concluiu que o diâmetro do coletor deveria permanecer grande bastante manter uma taxa alta de precipitação, o que tem que ser enfatizado ao se trabalhar com aspersores giratórios e de jatos descontínuos.

O objetivo deste trabalho foi desenvolver um coletor ideal, no formato e na adaptação ao ensaio, que possa vir a formar um circuito de coletores para testes de perfil de distribuição indoor, com manuseio para despejo da coleta de precipitação e retorno para a posição original. Buscou-se também aplicar uma automação no circuito que possa fornecer os dados em tempo real. Este circuito foi instalado no Laboratório de Ensaios em Equipamentos de Irrigação - LEEI, da Faculdade de Tecnologia CENTEC de Sobral laboratório acreditado pelo INMETRO para realizar ensaios em gotejadores, microaspersores e aspersores rotativos.

\section{MATERIAL E MÉTODOS}

Para ter um design econômico em termos de material e mão de obra, bem como facilitar a difusão desta tecnologia para outros centros de pesquisa, deduziu-se que a boca do coletor deveria ser construída com tubos de PVC branco (linha esgoto predial) de $200 \mathrm{~mm}$ de diâmetro nominal. O corpo (parte inferior), onde será armazenada á água coletada durante os ensaios, foi construído com tubos de PVC marrom (linha água fria) de $75 \mathrm{~mm}$ de diâmetro nominal. Para o fechamento do final do tubo marrom foi utilizado um CAP soldável (Figura 1). A parte maior do cone recebeu internamente a boca do coletor e a parte inferior do cone foi introduzida no depósito (Tubo PVC marrom) conforme detalhe da Figura 1. A parte do tubo de PVC $(200 \mathrm{~mm})$ que serviu de boca para o coletor teve sua borda biselada e, para fixação das peças de PVC ao cone de resina, utilizou-se uma cola do tipo Epóxi. Ressaltamos que como os tubos de PVC mudam o diâmetro interno e a espessura da parede conforme a marca é importante tomar o cuidado de utilizar a mesma marca para a confecção de todos os coletores. Os detalhes são mostrados na Figura 1.

Foi elaborado um software em linguagem Delphi para o gerenciamento automático do processo de ensaio dos aspersores e no momento da construção deste 


\section{COLETOR AUTOMÁTICO PARA ENSAIO DE ASPERSORES EM LABORATÓRIO (1) - DESENVOLVIMENTO DO MODELO}

programa foi destinado alguns campos de entrada de dados para consideração dos diâmetros reais dos tubos utilizados na confecção dos coletores.

Este software administra o acionamento da moto-bomba e o manuseio do circuito de coletores (despejar água e retornar a posição original) através de um pequeno motor acoplado a barra de aço onde ficam instalados os coletores. O programa também recebe as informações dos sensores de pressão e realizam a transformação em precipitação, além de elaborar os gráficos de perfil de distribuição.

Os detalhes e medidas das abraçadeiras de fixação do coletor ficaram a cargo do construtor em função do material disponível. A função da abraçadeira será de permitir o acoplamento de uma barra de aço transversal que possa unir todos os coletores do circuito e desta forma realizar um movimento que permita o despejo da água coletada e o retorno a posição original. No centro do CAP foi efetuado um furo de aproximadamente $4 \mathrm{~mm}$, no qual será inserido um micro tubo de polietileno para conexão com o sensor de pressão.

O sensor de pressão foi montado de modo que o seu centro coincida com o fundo do depósito (tubo marrom). $\mathrm{O}$ transdutor de pressão MPX5010DP é um sensor com capacidade de medida de pressão na faixa de 0 a $10 \mathrm{kPa}$ o que representa aproximadamente 1 metro de coluna de água (m.c.a.). Segundo o fabricante do sensor utilizado, o sinal de saída para o sensor sem carga é de $\approx 200 \mathrm{mV}$ e para a carga máxima é de $4700 \mathrm{mV}$. Portanto, a variação de tensão na saída do sensor é de 4500 $\mathrm{mV}$. Um Conversor Analógico Digital (A/D) de 10 bits retorna um valor de 0 count para uma tensão de $0 \mathrm{~V}$ e um valor de 1023 counts $\left(2^{10}\right.$ 1) para uma tensão de $5 \mathrm{~V}(5000 \mathrm{mV})$.
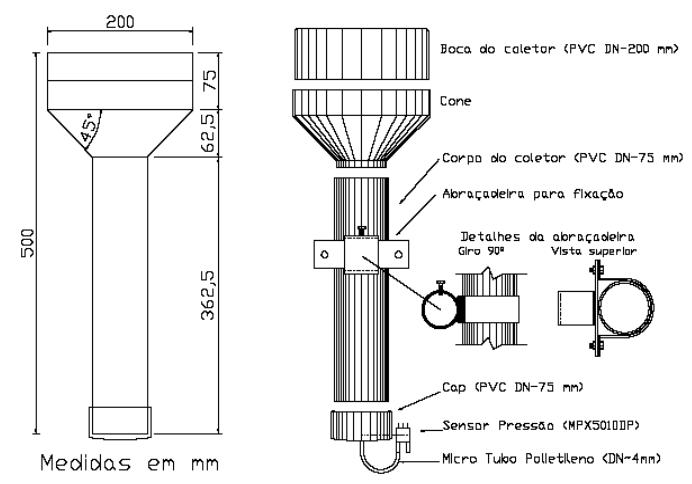

Figura 1 - Medidas sugeridas para o coletor e peças constituintes do coletor.

R. Bras. Agric. Irrigada v.2, n.1, p.24-28, 2008.

\section{RESULTADOS E DISCUSSÃO}

Hendawi et. al. (2002) propôs um coletor fabricado com ABS e policarbonato tratado contra UV, com uma altura total de $350 \mathrm{~mm}$ com a parte cilíndrica de $100 \mathrm{~mm}$ altura; diâmetro de $250 \mathrm{~mm}$; base cônica com um ângulo de inclinação de $55^{\circ}$ na altura de $210 \mathrm{~mm}$ e base de fixação na altura $50 \mathrm{~mm}$. O diâmetro interno foi de $42 \mathrm{~mm}$ com 4 cunhas verticais para ajuste. $\mathrm{O}$ coletor aqui proposto foi desenvolvido com o cone de conexão da boca com o depósito construído de resina com $2 \mathrm{~mm}$ de espessura para redução dos custos. A inclinação do cone foi de $45^{\circ}$, ao invés dos $55^{\circ}$ proposto por Hendawi et. al. (2002).

Considerando o diâmetro interno da boca do coletor (D), o diâmetro interno do depósito (d), a altura do depósito (H) e o tempo de funcionamento $(\mathrm{T})$, pode-se calcular a lâmina precipitada (L) e intensidade de precipitação (I) conforme mostrado a seguir.

A lâmina precipitada (L) pode ser calculada dividindo-se o volume acumulado dentro do depósito (V) pela área da seção transversal da boca do coletor (S).

$L=\frac{V}{S}$

em que:

L é lâmina precipitada, em m;

A intensidade de precipitação (I) pode ser calculada dividindo-se a lâmina precipitada (L) pelo tempo de coleta de água ou de precipitação efetiva.

$I=\frac{L}{T}$

em que:

T é o tempo de coleta de água, em hora;

I é a intensidade de precipitação, em $\mathrm{m} / \mathrm{h}$.

Multiplica-se a Equação 2 por 1000 para que o resultado seja convertido para a unidade padrão $(\mathrm{mm} / \mathrm{h})$. Associando todas as equações o cálculo da intensidade de precipitação pode ser simplificado.

$$
I=\frac{d^{2}}{D^{2}} H \frac{1}{T} 1000
$$




\section{COLETOR AUTOMÁTICO PARA ENSAIO DE ASPERSORES EM LABORATÓRIO (1) - DESENVOLVIMENTO DO MODELO \\ Considerando que o tempo de ensaio \\ CONCLUSÃO} deverá ser de uma hora, o termo T da Equação 3 pode ser subtraído, simplificando-se mais ainda a expressão matemática para o cálculo de I.

$$
I=\frac{d^{2}}{D^{2}} H 1000
$$

Em relação a automação, com os dois pares de valores obtidos dos dados do sensor que seriam $[(0 ; 0)$ e $(1023 ; 5000)]$ pôde-se ajustar uma equação linear e a partir desta, substituindo os valores $200 \mathrm{mV}$ e $4700 \mathrm{mV}$, encontrou-se os respectivos counts de 41 e 962. A diferença entre estes dois valores obtidos pela equação linear representa a faixa de variação da leitura do A/D para a faixa de variação de pressão do sensor, ou seja, 41 counts para 0 m.c.a. e 962 counts para 1 m.c.a.. Dividindo-se os 1000 mm.c.a. de um 1 m.c.a. pela diferença de counts (921) obtém-se a precisão absoluta dada pelo A/D de 10 bits operando com o sensor MPX5010DP. A precisão, portanto, será de 1,085 mm.c.a. (1000 mm.c.a. / 921 counts). Tomando-se como base os dados citados abaixo pode se estimar o valor máximo e mínimo possível de se medir com o coletor proposto. $\mathrm{D}$ $=200 \mathrm{~mm} ; \mathrm{d}=75 \mathrm{~mm} \mathrm{e} \mathrm{H}=362,5 \mathrm{~mm}$

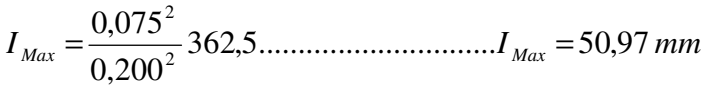

$$
\begin{aligned}
& I_{\text {Min }}=\frac{0,075^{2}}{0,200^{2}} 1,085 \ldots \ldots \ldots \ldots \ldots \ldots \ldots \ldots \ldots \ldots I_{\text {Min }}=0,1526 \mathrm{~mm}
\end{aligned}
$$

Este intervalo de valores é suficiente para se confeccionar um perfil de distribuição de água de um aspersor convencional de acordo com as especificações contidas em ABNT (2000).

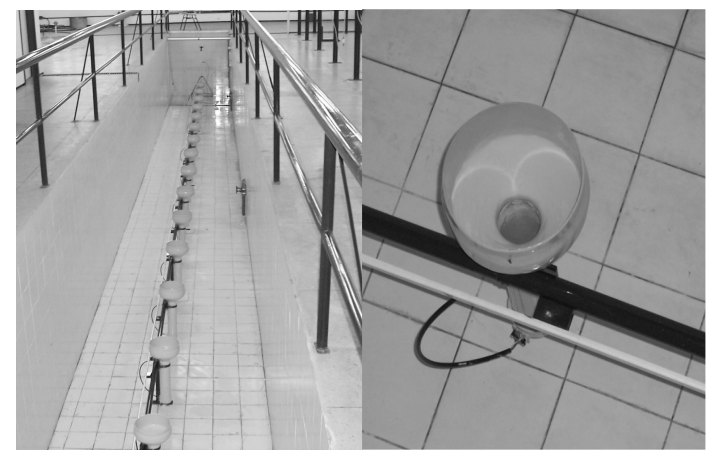

Figura 2 - Imagens do circuito de coletores e do coletor desenvolvido e instalado no LEEI.
O modelo de coletor desenvolvido possui plena capacidade de realizar o ensaio especificado, pois atende as condições de volume coletado, material econômico e é facilmente adaptável a uma automação economicamente viável.

A próxima etapa é a realização de testes para validar o modelo e identificar possíveis melhorias.

\section{AGRADECIMENTOS}

À FINEP e ao CNPq financiadores desta pesquisa.

\section{REFERÊNCIAS}

ASSOCIAÇÃO BRASILEIRA DE NORMAS TÉCNICAS - ABNT. NBR 7749-2. Equipamentos de irrigação agrícola Aspersores rotativos - Parte 2: Uniformidade de distribuição e métodos de ensaio. São Paulo, 2000.06p.

CARRAN P.S., A preliminary study of the efficiency of catch-cans used for irrigation sprinkler testing, internal Rapport ${ }^{\circ} \mathbf{7 8}$, New Zealand agricultural Engineering Institute, 1976.

FISCHER G.R., WALLENDER W.W., Collector size and test duration effects on sprinkler water distribution measurement, Transactions of the ASAE, v. 31, n. 4, p.538542. 1988

HENDAWI M., MOLLE B., FOLTON C., GRANIER J. Measurement Accuracy Analysis of Sprinkler Irrigation Rainfall in Relation to Collector Shape Journal of Irrigation and Drainage Engineering., V. 131, n. 5, p. 477483, 2005.

KOHL R.A., Sprinkler Precipitation Gage Errors, Transactions of the ASAE, v. 15, n. 1, p.264-271. 1972.

LIMA, S. C. R. V. Avaliação Hidráulica de Válvulas Reguladoras de Pressão Novas e com Diferentes Tempos de Utilização. 2001.83f. Dissertação (Mestrado em Irrigação e Drenagem) Universidade Federal do Ceará, Fortaleza.

LIVINGSTONE P., LOFTIS J.C., DUKE H.R., A wind tunnel study of sprinkler catch-can 
COLETOR AUTOMÁTICO PARA ENSAIO DE ASPERSORES EM LABORATÓRIO (1) DESENVOLVIMENTO DO MODELO

performance, Transactions of the ASAE, v.28, n.6, p.1961-1965, 1985.

MAREK T.H., SCHNEIDER A. D., BAKER S.M., POPHAM T.W., Accuracy of three sprinkler Collectors, Transactions of the ASAE, v.28, n.4, p.1191-1195, 1985.

MUELLER C.C., KIDDER E.H., Rain gage catch variation due to airflow disturbances around a standard rain gage, Water Resources Research, V. 8, n.4, p.1077-1082, 1972.

NEFF E.L., How much rain does a rain-gage gage, Journal of Hydrology, V.35, p.213-220, 1977. 